\title{
Review
}

\section{Cancer therapy in the necroptosis era}

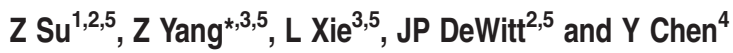

Necroptosis is a caspase-independent form of regulated cell death executed by the receptor-interacting protein kinase 1 (RIP1), RIP3, and mixed lineage kinase domain-like protein (MLKL). Recently, necroptosis-based cancer therapy has been proposed to be a novel strategy for antitumor treatment. However, a big controversy exists on whether this type of therapy is feasible or just a conceptual model. Proponents believe that because necroptosis and apoptosis use distinct molecular pathways, triggering necroptosis could be an alternative way to eradicate apoptosis-resistant cancer cells. This hypothesis has been preliminarily validated by recent studies. However, some skeptics doubt this strategy because of the intrinsic or acquired defects of necroptotic machinery observed in many cancer cells. Moreover, two other concerns are whether or not necroptosis inducers are selective in killing cancer cells without disturbing the normal cells and whether it will lead to inflammatory diseases. In this review, we summarize current studies surrounding this controversy on necroptosis-based antitumor research and discuss the advantages, potential issues, and countermeasures of this novel therapy. Cell Death and Differentiation (2016) 23, 748-756; doi:10.1038/cdd.2016.8; published online 26 February 2016

Facts

- A plethora of cancer cell lines can undergo necroptosis by classic necroptosis inducers and existing chemotherapeutic agents.

- Triggering necroptosis could be an alternative way to eradicate apoptosis-resistant cancer cells.

- Intrinsic or acquired defects of necroptotic machinery are observed in many cancer cells.

\section{Open Questions}

- What is the antitumor efficacy of necroptosis inducers in vivo?

- Can necroptosis inducers selectively trigger cell death in cancer cells and not normal cells?

- Can necroptosis-based cancer therapy result in inflammatory diseases?

- What is the role of necroptosis in radiation therapy?

Necrosis, a type of cell death resulting from disease, injury, or failure of the blood supply to an organ or tissue, was originally considered to be an accidental and unregulated cell death.
Accumulating evidence has shown that necrosis can be induced and proceed in a regulated manner like apoptosis, although in a caspase-independent manner. ${ }^{1}$ Necroptosis is a major and most well-studied form of regulated necrosis, but regulated necrosis may also include other forms, such as parthanatos, oxytosis, ferroptosis, NETosis, pyronecrosis, and pyroptosis. $^{2}$

Necroptosis can be induced by engaging with tumor necrosis factor (TNF) receptor superfamily, T-cell receptors, interferon receptors, Toll-like receptors, cellular metabolic and genotoxic stresses, or various anticancer compounds. ${ }^{3}$ The core necroptotic pathway is receptor-interacting protein kinase 1 (RIP1)-RIP3-mixed lineage kinase domain-like protein (MLKL). The RIP1-RIP3-MLKL complex, also called the 'necrosome', mediates the upstream cell death receptors (or other receptors) and downstream executing molecules and events such as reactive oxygen species (ROS) burst, plasma membrane permeabilization, and cytosolic ATP reduction. ${ }^{4}$ More specifically, RIP1 kinase activity is responsible for RIP3 phosphorylation, and subsequently, RIP3 phosphorylation further renders MLKL phosphorylation and trimerization. MLKL homotrimer then translocates to the plasma membrane and causes necrotic plasma membrane permeabilization, ${ }^{5}$ which serves as one of the necroptosisexecuting mechanisms. Alternatively, some necroptosis

${ }^{1}$ Department of Biochemistry and Molecular Biology, Medical School, Southeast University, Nanjing, Jiangsu 210009, China; ${ }^{2}$ Department of Cell Biology, Harvard Medical School, Boston, MA 02115, USA; ${ }^{3}$ Bone and Soft Tissue Tumors Research Center of Yunnan Province, Department of Orthopaedics, The Third Affiliated Hospital of Kunming Medical University (Tumor Hospital of Yunnan Province), Kunming, Yunnan 650118, China and ${ }^{4}$ Key Laboratory of Animal Models and Human Disease Mechanisms, Kunming Institute of Zoology, Chinese Academy of Sciences, Kunming, Yunnan 650223, China

${ }^{*}$ Corresponding author: Z Yang, Bone and Soft Tissue Tumors Research Center of Yunnan Province, Department of Orthopaedics, The Third Affiliated Hospital of Kunming Medical University (Tumor Hospital of Yunnan Province), Kunming, Yunnan 650118, China. Tel: +86 871 68221157; Fax: +86 871 68221157;

E-mail: yangzuozhang@163.com

${ }^{5}$ These authors contributed equally to this work and should be considered as co-first authors.

Abbreviations: RIP1, receptor-interacting protein kinase 1; RIP3, receptor-interacting protein kinase 3; MLKL, mixed lineage kinase domain-like protein; TNF, tumor necrosis factor; ROS, reactive oxygen species; cIAP1, cellular inhibitor of apoptosis 1; CYLD, deubiquitinase cylindromatosis; TRAF2, TNF receptor-associated factor 2; MDR, multidrug resistance; CLL, chronic lymphocytic leukemia; ALL, acute lymphoblastic leukemia; Nec-1, necrostatin-1; NSA, necrosulfonamide; DMF, dimethyl fumarate; TRAIL, TNF-related apoptosis-inducing ligand; HVJ-E, hemagglutinating virus of Japan-envelope

Received 24.10.15; revised 21.12.15; accepted 03.1.16; Edited by H Steller; published online 26.2.16 
inducers may bypass RIP1 to activate directly RIP3 or MLKL. ${ }^{6}$ Necroptosis can be pharmacologically inhibited by chemical compounds, such as necrostatin-1 (Nec-1; RIP1 kinase inhibitor), GSK-843/-872/-840 (RIP3 kinase inhibitor), and necrosulfonamide (MLKL inhibitor). ${ }^{4}$

The most well-studied necroptotic cell death is TNF-mediated necroptosis. In this model, TNF binds to the TNF receptors on the plasma membrane and engages in a membrane-associated complex formation (i.e. Complex I) that includes E3 ligases cellular inhibitor of apoptosis 1 (clAP1) and cIAP2, deubiquitinase cylindromatosis (CYLD), RIP1, and TNF receptor-associated factor 2 (TRAF2). clAPs induce RIP1 polyubiquitination to inhibit the formation of Complex Ila (i.e. caspase-8, Fas-associated death domain (FADD), and RIP1) and Complex Ilb (i.e. caspase-8, FADD, RIP1, RIP3, and MLKL), thus blocking RIP1-mediated apoptosis or necroptosis. In contrast, CYLD deubiquitinates RIP1 and promotes the packaging of Complexes Ila and IIb. ${ }^{1}$ Furthermore, in Complex Ila, activated caspase-8 cleaves and halts the activities of RIP1, RIP3, and CYLD. Cleaved RIP1 and RIP3 lose their transphosphorylation and downstream substrate phosphorylation capabilities. However, when the cleavage of RIP1 and RIP3 is prevented by caspase-8 inhibitors (e.g. zVAD) or by the genetic deletion of caspase- 8 or FADD, Complex IIb is formed and necroptosis is initiated. ${ }^{1,7}$ Necroptotic pathway is schematically diagrammed in Figure 1.

Unlike apoptosis, necroptosis is not normally detected during embryogenesis; however, the abnormal activation of necroptosis was reported to affect normal development and lymphocyte homeostasis. ${ }^{8-13}$ Necroptosis may also serve a protective role in virus infections by restricting virus propagation or increasing inflammatory response. ${ }^{14,15}$ In addition, necroptosis has been implicated in the pathogenesis of a variety of human diseases, including TNF-mediated hypothermia and systemic inflammation, ischemic reperfusion injury, neurodegeneration, Gaucher's disease, progressive atherosclerotic lesions, and cancer. ${ }^{16}$ The major differences between apoptosis and necroptosis are summarized in Table 1.

\section{What Types of Cancers Have the Necroptotic Machinery?}

In the past few years, a plethora of cancer cell lines that undergo necroptosis by classic necroptosis inducers (e.g. TNF+cycloheximide+ZVAD, TNF+IAP inhibitors+zVAD, and TNF+5Z-7-oxozeaenol+zVAD) or existing chemotherapeutic agents has been validated. These cancer cell lines cover almost all common cancer types, including colorectal cancer, leukemia, multiple myeloma, lung cancer, ovarian cancer, breast cancer, hepatocarcinoma, bladder carcinoma, head and neck carcinoma, glioblastoma, cervical cancer, and neuroblastoma. Interestingly, among these cancer cells, colorectal cancer cells and hematopoietic neoplasms (e.g. leukemia and multiple myeloma) cells seem to be more sensitive and responsive to necroptosis inducers. Colorectal cancer cells (i.e. HT29, Caco-2, and CT26) can undergo necroptosis upon stimulation with TNF+IAP inhibitors+zVAD, TRAIL (TNF-related-apoptosis-inducing-ligand) alone (in acidic $\mathrm{pH}$ ) or upon combination with cycloheximide/ homoharringtonine and zVAD, 5-fluorouracil+pancaspase inhibitors (zVAD or IDN-7314), hypoxia, poly I:C+ZVAD, and dimethyl fumarate..$^{5,17-24}$ Jurkat and CEM-C1, lymphoblastic leukemia cell lines, and MV4-11, Molm13, K562, and U937, myeloid leukemia cell lines, were reported to undergo necroptosis upon induction with anti-FAS antibody+cycloheximide+zVAD, 24(S)-hydroxycholesterol+zVAD/caspase-8 deficiency, obatoclax, obatoclax+dexamethasone, Smac mimetics+5-aza-2'-deoxycytidine, Smac mimetics+cytarabine $+z V A D$, shikonin and its analogs, TNF+Smac mimetics $+z V A D$, TRAIL+ZVAD, and staurosporine+zVAD. ${ }^{5,25-31}$ Furthermore, KMS-12-PE, RPMI-8226, and U266, myeloma cell lines undergo necroptosis upon treatment with shikonin in the presence or absence of zVAD. ${ }^{32}$ The cancer cells that can undergo necroptosis, their inducers, and evidence are summarized in Table 2.

Although many cancer cell lines are reported to have necroptotic machinery, they comprise a small number of cancer cell family. Numerous cancer cell lines, such as HeLa cells, colorectal cancer cells (HCT-116), and human ovarian cancer cells (OVCAR4) without an effective necroptotic machinery are unsuitable necroptotic-based cancer therapy targets. ${ }^{17,33,34}$ Furthermore, necroptosis-sensitive cells are only responsive to a few specific necroptosis inducers but not to all necroptosis inducers. For example, A549 cells undergo necroptosis upon some specific chemical compounds (see Table 1) but are irresponsive to classic necroptosis inducers, TNF+cycloheximide+zVAD, TNF+Smac mimetics+zVAD, and TNF+5Z-7-oxozeaenol+zVAD. So far, few histological or animal data show whether these inducers can trigger tumor necroptosis in vivo.

\section{Triggering Necroptosis Is a Promising Strategy to Overcome Apoptosis Resistance in Cancer}

Proapoptotic therapy (e.g., using cisplatin, carboplatin, paclitaxel, 5-fluorouracil, and gemcitabine), a major form of chemotherapy, is the principal method for cancer treatment, but the effectiveness of this therapy is limited by drug resistance. Drug resistance may result from disrupted apoptosis machinery, overactivated prosurvival signaling pathways, increased expression of the therapeutic target, activation of alternative compensatory pathways, high degree of molecular heterogeneity in tumor cells, upregulation of drug transporters and multidrug resistance. ${ }^{35}$ Among these findings, apoptosis dysfunction appears to be a critical factor in intrinsic and acquired chemotherapy drug resistance. Genetic mutations and gene abnormal expression are prevalent in extrinsic and intrinsic apoptotic pathways in cancer cells; previous studies have shown increased expressions of antiapoptotic proteins FLIP, Bcl-2, $\mathrm{Bcl}-\mathrm{xL}$, or $\mathrm{Mcl}-1$ and mutations in p53, Apaf-1, Bax, Fas, FADD, or caspases. ${ }^{35,36}$

Because necroptotic pathway uses components that are different from apoptotic pathway, cancer cells that are resistant to apoptosis agents may be sensitive to necroptosis inducers. In other words, apoptosis needs activation of caspases, but necroptosis can terminate cancer cells when caspases are inhibited or defective. Many research groups have validated this hypothesis. Han et al. ${ }^{36}$ reported that shikonin, a naturally occurring naphthoquinone, induced necroptotic cell death in $\mathrm{Bcl}-2$ - or Bcl-xL-overexpressed MCF-7 breast cancer cells that 


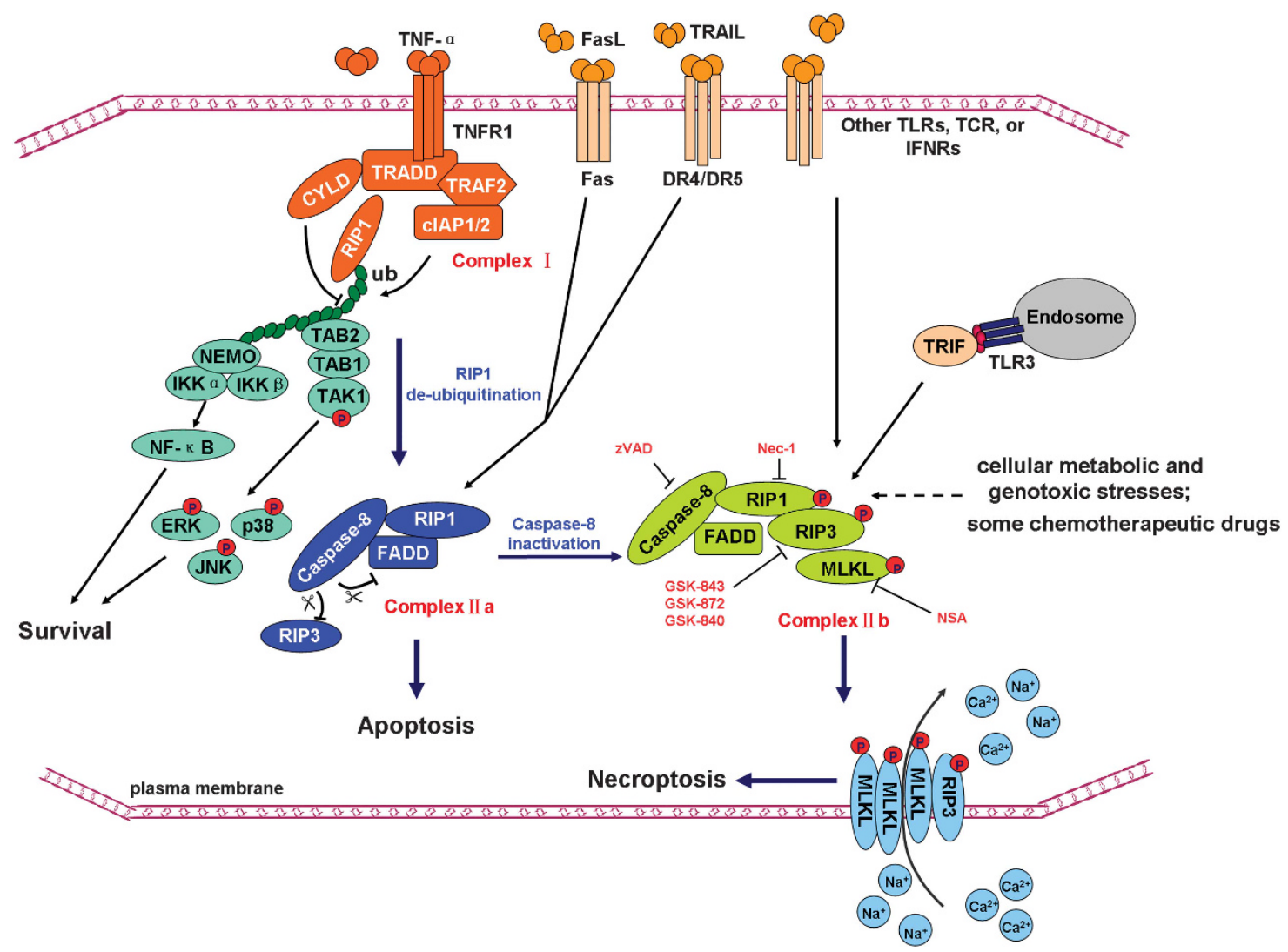

Figure 1 Necroptotic pathway. Necroptosis can be triggered by engagement of TNF- $\alpha$ to TNF receptor superfamily (e.g. TNFR, Fas, and DR4/DR5), Toll-like receptors (e.g. TLR3 and TLR4), T-cell receptors, interferon receptors, cellular metabolic and genotoxic stresses, or some anticancer compounds. RIP1-RIP3-MLKL complex, also called 'necrosome', is a critical mediator of the necroptotic pathway that bridges the signals of upstream cell death receptors (or other receptors) and downstream executing molecules and events, such as plasma membrane permeabilization, cytosolic ATP reduction, and reactive oxygen species (ROS) burst. In TNF- $\alpha$-induced necroptosis, TNF binds to the TNF receptors and engages a big complex formation called Complex I, which includes cIAP1, cIAP2, CYLD, RIP1, and TRAF2. cIAPs induce RIP1 polyubiquitination to inhibit Complex Ila in which caspase-8, FADD, and RIP1 are involved, and Complex Illb, comprising the caspase-8, FADD, RIP1, RIP3, MLKL formation, thus blocking RIP1-mediated apoptosis or necroptosis. In addition, polyubiquitinated RIP1 recruits NEMO and TAB2 that mediate NF- $k B$ activation and MAPKs (i.e. ERK, JNK, p38) activation, respectively. Unlike the RIP1 polyubiquitination that benefit cell survival, CYLD deubiquitinates RIP1 and promotes the packaging of Complexes Ila and IIb. In the Complex Ila, activated caspase-8 cleaves and abolishes the activities of RIP1, RIP3, and CYLD, thus blocking necroptosis. However, when the cleavage of RIP1 and RIP3 is prevented by caspase-8 inhibitors (e.g., zVAD) or by the genetic deletion of caspase-8 or FADD, the Complex llb forms and initiates necroptosis. Necroptosis can be pharmacologically inhibited by Nec-1 (RIP1 kinase inhibitor), GSK-843/-872 (RIP3 kinase inhibitor), and necrosulfonamide (NSA, MLKL inhibitor). TNFR1, TNF receptor 1; NEMO, IKK- $\gamma$; DR4/5, death receptor 4 or 5; TLRs, toll-like receptors; TCR, T-cell receptor; TAK1, transforming growth factor- $\beta$-activated kinase 1; TAB2, TAK1-binding protein 2; TRIF, Toll/LL-1 receptor domain-containing adaptor-inducing interferon- $\beta$

are resistant to proapoptosis drugs. Interestingly, shikonin also triggered necroptotic cell death in MCF-7/Adr cells that are characterized by overexpression of a multidrug resistance protein P-glycoprotein and high resistance to a panel of anticancer drugs including anthracyclines, taxanes, and vinca alkaloids.

Glucocorticoids were among the first drug classes used in the treatment of patients with acute lymphoblastic leukemia (ALL) by inducing cell-cycle arrest and apoptosis. However, a group of ALL patients showed poor response to glucocorticoids, which is a strong predictor of adverse outcome for ALL. Bonapace et al. ${ }^{27}$ reported that obatoclax, a putative antagonist of $\mathrm{Bcl}-2$ family members, triggered autophagydependent necroptosis to reverse glucocorticoids resistance in ALL cells. Another group showed that combination of a Smac mimetic BV6 and TNF triggered necroptotic cell death in FADD-deficient, caspase-8-deficient, or caspase-8-deficient and $\mathrm{Bcl}-2$-overexpressing variants of human Jurkat leukemia cells that are resistant to apopotosis inducers. ${ }^{37}$ Ovarian cancer patients are typically treated with carboplatin and paclitaxel. Although many tumors initially respond to treatment, $60-85 \%$ of patients experience disease recurrence following primary therapy because of acquired resistance to these proapoptosis drugs. ${ }^{33}$ McCabe et al. ${ }^{33}$ reported that IAP antagonist with caspase inhibitor ZVAD treatment induced TNF-dependent necroptotic death in several cisplatin and IAP antagonist-resistant ovarian carcinoma cell lines and some cisplatin-resistant patient-derived cancer cells.

Resistance to the proapoptotic drug 5-fluorouracil is a major challenge in the adjuvant chemotherapy of colorectal cancer. Metzig et $a l .{ }^{24}$ reported that the usage of pancaspase inhibitors sensitized resistant colorectal cancer cells to 5-fluorouracil by triggering necroptosis. In vivo xenograft experiments showed that a novel pancaspase inhibitor IDN-7314 in combination with 5-fluorouracil synergistically blocked tumor growth compared with 5-fluorouracil alone. 
Table 1 Comparison of apoptosis and necroptosis ${ }^{49,54}$

\begin{tabular}{|c|c|c|}
\hline Features & Apoptosis & Necroptosis (a major form of regulated necrosis) \\
\hline Cytoplasmic shrinkage & Yes & No \\
\hline Chromatin condensation & Yes & Mild \\
\hline Nuclear fragmentation & Yes & No \\
\hline Membrane blebbing & Yes & No \\
\hline $\begin{array}{l}\text { Shedding of apoptotic } \\
\text { bodies }\end{array}$ & Yes & No \\
\hline Swelling of organelles & No & Yes \\
\hline $\begin{array}{l}\text { Lysosomal membrane } \\
\text { permeabilization }\end{array}$ & No & Yes \\
\hline $\begin{array}{l}\text { Plasma membrane } \\
\text { permeabilization }\end{array}$ & No & Yes \\
\hline Caspase activation & Yes & No \\
\hline Key regulators in pathway & $\begin{array}{l}\text { Bid, Bax/Bak; cytochrome } c \text {; Apaf-1; caspase-9; } \\
\text { caspase-8/10; FADD; RIP1 }\end{array}$ & RIP1, RIP3, MLKL \\
\hline $\begin{array}{l}\text { Executors of cell death } \\
\text { Inhibitors }\end{array}$ & $\begin{array}{l}\text { Caspase-3; caspase-7 } \\
\text { zVAD }\end{array}$ & $\begin{array}{l}\text { MLKL trimer and ion channels formation } \\
\text { Nec-1 (RIP1 kinase inhibitor); GSK-843, GSK-872 and } \\
\text { GSK-840 (RIP3 kinase inhibitors); NSA (MLKL inhibitor) }\end{array}$ \\
\hline $\begin{array}{l}\text { Physiological and patholo- } \\
\text { gical roles }\end{array}$ & $\begin{array}{l}\text { Controlling cell numbers during embryogenesis and } \\
\text { homeostasis, immune regulation, and pathogen } \\
\text { defense. Inhibition of apoptosis may result in cancers, } \\
\text { autoimmune diseases, inflammatory diseases, and } \\
\text { viral infections }\end{array}$ & $\begin{array}{l}\text { Virus infection, TNF-mediated hypothermia and systemic } \\
\text { inflammation, ischemic reperfusion injury, neurodegen- } \\
\text { eration, Gaucher's disease, progressive atherosclerotic } \\
\text { lesions, and cancers }\end{array}$ \\
\hline
\end{tabular}

Abbreviations: Nec-1, necrostatin-1; NSA, necrosulfonamide

Note: Necroptosis is a major and most well-studied form of regulated necrosis, but regulated necrosis may also include other forms, such as parthanatos, oxytosis, ferroptosis, NETosis, pyronecrosis, and pyroptosis ${ }^{2}$

Notably, pretreatment with zVAD made $>50 \%$ fresh sliced tumor specimens of colorectal cancer patients produce more cell death in response to 5-fluorouracil. ${ }^{24}$ This indicates that a subgroup of patients could benefit from pronecroptosis treatment.

For a patient experiencing resistance to one or more traditional chemotherapeutic drugs, they may consider using different combinations of chemotherapeutic drugs or changing to other therapies using molecular targeted drugs (e.g., tyrosine kinase inhibitors and programmed death-1 inhibitors). However, pronecroptosis therapy will be another choice available to conquer drug resistance.

\section{How Do Cancer Cells Evade Necroptosis and How Can We Achieve Successful Necroptosis-based Therapy?}

How do cancer cells evade necroptosis? As mentioned above, some cancer cells can undergo necroptosis, and other cancer cells display resistance to necroptosis inducers. There are two major reasons for the failure of necroptosis machinery:

1. Cancer cells may eradicate necroptosis machinery by downregulation and functional mutations of RIP1, RIP3, and MLKL: According to the study by Moriwaki et al., ${ }^{17}$ RIP1 mRNA and protein expressions are decreased in only a few cancer cells, whereas RIP3 mRNA and protein expressions are downregulated in numerous cancer cells. HeLa cell is a well-known necroptosis resistance cancer cell line, which expresses a normal level of RIP1 but a low level of RIP3. However, when RIP3 gene is ectopically expressed in HeLa cells, they become sensitive to commonly used necroptosis inducers. $^{34}$ In vivo data showed that RIP1 and RIP3 expressions were downregulated in primary colon cancer tissues compared with those in normal adjacent tissues. ${ }^{17}$ This finding is in accordance with another study showing a significant decrease of RIP3 expression in most acute myeloid leukemia samples without a significant decrease in the expression of RIP1. ${ }^{38}$ Liu et al. ${ }^{39}$ reported that two key components of necroptotic machinery, RIP3 and CYLD, were markedly downregulated in chronic lymphocytic leukemia (CLL), which could be responsible for the failure of TNF with zVAD-induced necroptosis in CLL. Moreover, a pancreatic adenocarcinoma study showed that reduced expression of MLKL was associated with decreased overall survival $(6$ months versus 17 months; $P=0.006)$. In a subset of patients who received adjuvant chemotherapy, low MLKL expression was associated with decreased recurrence-free survival (5 months versus 15 months; $P=0.006$ ) and decreased overall survival (6 months versus 19 months; $P<0.0001) .{ }^{40}$ A microarray study indicated that the frequency of hypermethylation in promoter region was high for RIP3 in small-cell carcinoma (57\%), which resulted in the loss of RIP3 expression. ${ }^{41}$

According to the COSMIC database, ${ }^{42}$ amino-acid mutations for RIP1, RIP3, and MLKL have been observed in human cancer tissues, which may attenuate the RIP kinase activity or change the interaction with other proteins, leading to the disruption of necroptosis machinery. In non-Hodgkin lymphoma, singlenucleotide polymorphisms in the RIP3 gene were detected in 458 patients and were correlated with increased risk of nonHodgkin lymphoma, which suggests that genetic variations in the RIP3 gene may contribute to the onset of this disease. ${ }^{43}$

Taken together, these studies suggest that RIP1-RIP3MLKL-dependent necroptosis machinery may be a possible mechanism to restrict tumorigenesis and progression. However, tumor cells have evolved a series of strategies to evade necroptosis-induced cell death through downregulation of RIP1, RIP3, and MLKL or functional mutations on these proteins. 
Table 2 Cancer cells that can undergo necroptosis

\begin{tabular}{lll}
\hline Cancer cell lines & Cell type & Necroptosis inducers \\
\hline HT29 & $\begin{array}{l}\text { Human colorectal } \\
\text { adenocarcinoma cells }\end{array}$ & \\
& \\
& TRAIL in acidic condition \\
& \\
& \\
& \\
& TRAIL+cycloheximide+zVAD; TRAIL \\
& +homoharringtonine+zVAD; \\
& 5-Fluorouracil+pancaspase inhibitors \\
& (zVAD or IDN-7314)
\end{tabular}

\section{Caco-2}

CT26

CEM-C1

MV4-11; Molm13

K562

U937

KMS-12-PE; RPMI-8226; U266

A549

OVCAR3

$\mathrm{HaCaT}$

MCF-7
Human colonic carcinoma cells Mouse colon carcinoma cells

Hypoxia

Poly I:C+zVAD

DMF

Human acute T-cell leukemia cells

Human acute $\mathrm{T}$ leukemia cells

Human acute myeloid leukemia cells

Human erythromyeloblastoid leukemia cells

Human myelomonocytic cells

Human myeloma cells

Shikonin; shikonin+zVAD

Human lung carcinoma cells

FTY720 (a sphingolipid analog drug)

Rhenium (V) oxo complexes

Chalcone derivative chalcone-24 (Chal24)

Human ovarian cancer cells

IAPa (an IAP antagonist)+ZVAD

Murine fibrosarcoma cells

Human immortalized keratinocytes

Human breast adenocarcinoma cells

Anti-FAS antibody+cycloheximide+zVAD hydroxycholesterol+ caspase-8 deficiency obatoclax (a small-molecule inhibitor of antiapoptotic $\mathrm{Bcl}-2$ proteins) +dexamethasone tidine (a DNA methyltransferase inhibitor) $+\mathrm{ZVAD}$

BV6+cytarabine+zVAD

Shikonin and its analogs

$\mathrm{TNF}+\mathrm{Smac}$ mimetics+zVAD

TRAIL+zVAD; staurosporine+zVAD

TNF; TNF+BV6; TNF+5Z-7-oxozeaenol (a TAK1 inhibitor) zVAD

TRAIL+ZVAD+TRAF2 knockdown; FcFasL+ zVAD+TRAF2 knockdown; TRAIL +TWEAK+zVAD; TRAIL+BV6+zVAD Sodium nitroprusside; TNF- $a+z V A D$ +either cycloheximide or Smac mimetic

\section{References and evidence for necroptosis}

Cai et al..$^{5} \mathrm{MLKL}$ trimer; RIP3 knockdown or knockout; Nec-1 protection

Moriwaki et al. ${ }^{17} \mathrm{Nec}-1$ or GSK-840 (a RIP3 kinase inihibitor) protection; RIP1 or RIP3 knockdown

Meurette et al. ${ }^{18}$ electron microscope imaging; Hoechst/PI double staining; using RIP1 kinase dead cells

Jouan-Lanhouet et al. ${ }^{19} \mathrm{Nec}-1$ protection; RIP1 or RIP3 knockdown and knockout Philipp et al. ${ }^{20}$ RIP3 smear bands; Nec-1 and NSA protection; phosphorylation of MLKL; RIP1, RIP3, or MLKL knockdown Metzig et al. ${ }^{24} \mathrm{Nec}-1$ protection; RIP1, RIP3, and MLKL knockdown; phosphorylation of MLKL

Huang et al. ${ }^{21} \mathrm{Nec}-1$ protection; RIP1 knockdown; RIP1-RIP3 necrosome formation Takemura et al. $^{22} \mathrm{Nec}-1$ protection; ROS scavenger protection; RIP3 knockdown; RIP1-RIP3 necrosome formation

Xie et al. ${ }^{23} \mathrm{Nec}-1$ protection; electron microscope imaging (a disrupted cell membrane, mitochondrial swelling, reticulum expansion, etc.)

24(S)-hydroxycholesterol+zVAD or 24(S)-

BV6 (Smac mimetic)+ 5-aza-2'-deoxycy-

Degterev et al. ${ }^{25} \mathrm{Nec}-1, \mathrm{Nec}-3$, and $\mathrm{Nec}-5$ protection; RIP1 autophosphorylation Yamanaka et al. ${ }^{26} \mathrm{Nec}-1$ protection; RIP3 knockdown

Bonapace et al. ${ }^{27}$ Not rescued by zVAD; Nec-1 protection; electron microscope imaging; RIP1 knockout; CYLD knockdown

Bonapace et al. ${ }^{27}$ Not rescued by zVAD; Nec-1 protection

Steinhart et al. ${ }^{28} \mathrm{Nec}-1$ protection; NSA protection

Chromik et al. ${ }^{29} \mathrm{Nec}-1$ protection; NSA protection

Xuan et al. ${ }^{30} \mathrm{Nec}-1$ protection; electron microscopes imaging

Cai et al. ${ }^{5} \mathrm{MLKL}$ trimer

Dunai et al. ${ }^{31} \mathrm{Nec}-1$ protection; NSA protection; Hoechst/PI double staining

Wada et al. ${ }^{32}$ Low concentration of shikonin induces apoptosis (caspases cleavage; zVAD protects cell death); high concentration of shikonin induces necroptosis (less caspases cleavage; Nec-1 but not ZVAD protects cell death)

Saddoughi et al. ${ }^{55} \mathrm{Nec}-1$ protection; RIP1 knockdown

Suntharalingam et al. ${ }^{56} \mathrm{Nec}-1$ protection; ROS production; Hoechst/PI double staining He et al. ${ }^{57} \mathrm{Nec}-1$ protection; RIP1 and RIP3 knockdown

McCabe et al. ${ }^{33} \mathrm{Nec}-1$ protection; NSA protection; RIP1-RIP3 necrosome formation; MLKL phosphorylation; RIP3 knockdown Vanlangenakker et al. ${ }^{58} \mathrm{Nec}-1$ protection; RIP3

or CYLD knockdown; necrosome formation Hitomi et al. ${ }^{59} \mathrm{Nec}-1$ protection; RIP1 knockdown

Karl et al. ${ }^{60} \mathrm{Nec}-1$ protection; using RIP3 deficient and overexpressed cells

Kim et al. ${ }^{61} \mathrm{Nec}-1$ or dabrafenib (a RIP3 inhibitor) protection; RIP3 knockdown or overexpression; MLKL phosphorylation Han et al. ${ }^{36}$ electron microscope imaging (swollen mitochondria, plasma membrane rupture, autophagosomes, autophagic 
Table 2 Continued

\begin{tabular}{|c|c|c|c|}
\hline Cancer cell lines & Cell type & Necroptosis inducers & References and evidence for necroptosis \\
\hline & & & $\begin{array}{l}\text { vacuoles containing membranous whorls, } \\
\text { severe damage of mitochondria with disrupted } \\
\text { internal structures). Nec-1 protection; zVAD } \\
\text { does not protect cell death }\end{array}$ \\
\hline MDA-MB-231 & $\begin{array}{l}\text { Human breast adenocarci- } \\
\text { noma cells }\end{array}$ & $\begin{array}{l}\text { 3-Bromopyruvate (an inhibitor of hexoki- } \\
\text { nase II)+ chloroquine }\end{array}$ & $\begin{array}{l}\text { Zhang et al. }{ }^{48} \text { RIP1 or RIP3 knockdown; Nec-1 } \\
\text { protection; zVAD does not protect cell death }\end{array}$ \\
\hline HepG2 & $\begin{array}{l}\text { Human hepatocarcinoma } \\
\text { cells }\end{array}$ & TRAIL in acidic condition & $\begin{array}{l}\text { Jouan-Lanhouet et al. }{ }^{19} \text { RIP1 or RIP3 } \\
\text { kncokdown }\end{array}$ \\
\hline Mz-ChA-1 & $\begin{array}{l}\text { Human gallbladder adenocar- } \\
\text { cinoma cells }\end{array}$ & $\begin{array}{l}\text { TRAIL+cycloheximide+zVAD; TRAIL } \\
\text { +homoharringtonine+zVAD }\end{array}$ & $\begin{array}{l}\text { Philipp et al. }{ }^{20} \text { RIP3 smear bands; Nec-1 and } \\
\text { NSA protection; phosphorylation of MLKL; } \\
\text { RIP1, RIP3, or MLKL knockdown }\end{array}$ \\
\hline UM-UC-3 & $\begin{array}{l}\text { Human bladder carcinoma } \\
\text { cells }\end{array}$ & Chal-24 & He et al. ${ }^{57}$ RIP1 and RIP3 knockdown \\
\hline RMS13 & $\begin{array}{l}\text { Human rhabdomyosarcoma } \\
\text { cells }\end{array}$ & Obatoclax & $\begin{array}{l}\text { Basit et al. }{ }^{47} \text { zVAD does not protect cell death; } \\
\text { RIP1-RIP3 necrosome formation; Nec-1 } \\
\text { protection; RIP1 or RIP3 knockdown }\end{array}$ \\
\hline $\begin{array}{l}\text { SCC-11 } \\
\text { (JHU-029); } \\
\text { SCC-25 }\end{array}$ & $\begin{array}{l}\text { Human head and neck } \\
\text { squamous cell carcinoma } \\
\text { cells }\end{array}$ & Cisplatin & $\begin{array}{l}\text { Ratovitski et al. }{ }^{62} \mathrm{Nec}-1 \text { protection; NSA } \\
\text { protection; RIP1 silencing }\end{array}$ \\
\hline C666-1; HK-1 & $\begin{array}{l}\text { Human nasopharyngeal car- } \\
\text { cinoma cells }\end{array}$ & $\begin{array}{l}\text { Neoalbaconol (a constituent extracted } \\
\text { from Albatrellus confluens) }\end{array}$ & $\begin{array}{l}\text { Yu et al. }{ }^{63} \mathrm{MLKL} \text { phosphorylation; RIP1 or } \\
\text { RIP3 knockdown; Nec-1 but not zVAD } \\
\text { protection }\end{array}$ \\
\hline C4-I & $\begin{array}{l}\text { Human cervical squamous } \\
\text { cell carcinoma cells }\end{array}$ & Poly I:C; poly I:C+zVAD & Schmidt et al. ${ }^{46}$ RIP3 knockdown \\
\hline TE671 & Human medulloblastoma cells & Obatoclax & $\begin{array}{l}\text { Basit et al. }{ }^{47} \text { zVAD does not protect cell death; } \\
\text { RIP1-RIP3 necrosome formation; Nec-1 } \\
\text { protection; RIP1 or RIP3 knockdown }\end{array}$ \\
\hline \multirow{2}{*}{$\begin{array}{l}\text { SK-N-SH; SK-N- } \\
\text { AS } \\
\text { LN18 cell }\end{array}$} & Human neuroblastoma cells & \multirow{2}{*}{$\begin{array}{l}\text { Hemagglutinating virus of Japan- } \\
\text { envelope (HVJ-E) } \\
\text { 5-Aminolevulinic acid (a photosensitizer } \\
\text { precursor) based photodynamic therapy }\end{array}$} & $\begin{array}{l}\text { Nomura et al. }{ }^{64} \text { zVAD does not protect cell } \\
\text { death; Nec-1 protection; RIP1 phosphorylation }\end{array}$ \\
\hline & Human glioblastoma cells & & $\begin{array}{l}\text { Coupienne et al. }{ }^{65} \text { RIP1-RIP3 necrosome } \\
\text { formation; Nec-1 protection; RIP3 knockdown }\end{array}$ \\
\hline
\end{tabular}

Abbreviations: DMF, dimethyl fumarate; HVJ-E, hemagglutinating virus of Japan-envelope; zVAD, pancaspase inhibitor; TRAIL, TNF-related apoptosis-inducing ligand; TWEAK, TNF-like weak inducer of apoptosis; DMF, dimethyl fumarate; obatoclax, a small-molecule inhibitor of antiapoptotic Bcl-2 proteins; BV6, a Smac mimetic (IAP antagonist); 5-aza-2'-deoxycytidine, a DNA methyltransferase inhibitor; FTY720, a sphingolipid analog drug; Chal-24, chalcone derivative chalcone-24; 5Z-7-oxozeaenol, a TAK1 inhibitor; 3-bromopyruvate, an inhibitor of hexokinase II; neoalbaconol, a constituent extracted from Albatrellus confluens; HVJ-E, hemagglutinating virus of Japan-envelope; 5-aminolevulinic acid, a photosensitizer precursor; Nec-1, necrostatin-1 (a RIP1 kinase inhibitor); GSK-840, a RIP3 kinase inihibior; NSA, necrosulfonamide (an MLKL inhibitor); DMF, dimethyl fumarate; HVJ-E, hemagglutinating virus of Japan-envelope

2. Hypoxia-induced overactive anaerobic glycolytic metabolism and reduced RIP1/RIP3 expression confer resistance to necroptosis: In tumor progression, hypoxia, a common phenomenon in solid tumors because of rapid growth and poor vascularization, leads to necrosis inside the tumor, but conversely enhances metabolic reprogramming, angiogenesis, and metastasis of tumor. ${ }^{44}$ In a hypoxic condition, cancer cells may undergo classic necroptotic events, such as the RIP1/RIP3 complex formation and phosphorylation, plasma membrane disintegration, and protection by necrostatin-1. ${ }^{21}$ However, cancer cells can survive under the hypoxic condition by reprogramming metabolic pathway to greatly enhance anaerobic glycolysis. One possible reason is that glycolytic metabolism confers resistance to RIP1/RIP3-dependent necroptosis partly through scavenging of mitochondrial free radicals by metabolic product pyruvate. ${ }^{21}$

In addition, according to Moriwaki et al., ${ }^{17}$ RIP1 and RIP3 expression in mRNA and protein levels were reduced in some colon carcinoma cell lines with exposure to hypoxia for $6 \mathrm{~h}$ or longer by significantly lowering the sensitivity to TNF, IAP inhibitor and zVAD-induced necroptosis.

\section{How to achieve a successful necroptosis-based therapy?}

1. Conduct a genetic detection of RIP1, RIP3, and MLKL before using a pronecroptotic drug on patients: Similar to molecular targeted therapies, the first step for the future of necroptosis-based cancer therapy is to do the necroptotic genes and proteins detections for RIP1, RIP3, and MLKL. Through these detections, the presence of genetic mutations at key loci of these genes, which may affect their functions and the expression levels of these proteins in tumor tissues, will be determined. If the necroptotic machinery is abnormal or damaged, necroptosis-based therapy is not a viable option.

2. Combination with other therapeutic strategies to sensitize cancer cells to necroptosis-based therapy: As discussed above, hypoxia attenuated necroptosis by either downregulating RIP1 and RIP3 or reprogramming glycolytic metabolism; therefore, necroptosis-based therapy in combination with drugs targeting hypoxia (e.g. tirapazamine and antagonists of hypoxia-inducible factor 1 ) or in combination with inhibitors (e.g., iodoacetate) of glyceraldehyde-3-phosphate dehydrogenase, a key enzyme in anaerobic glycolysis, could be more effective.

Moreover, Mouratidis et al. ${ }^{45}$ reported that RIP3 protein levels were increased after exposure of human colon cancer cells to a thermal dose. This suggests that heat therapy may synergize with necroptosis-based therapy as an effective future cancer treatment.

3. Develop pronecroptotic drugs that directly target MLKL: The classic necroptotic pathway is from RIP1 to RIP3 and to MLKL, 


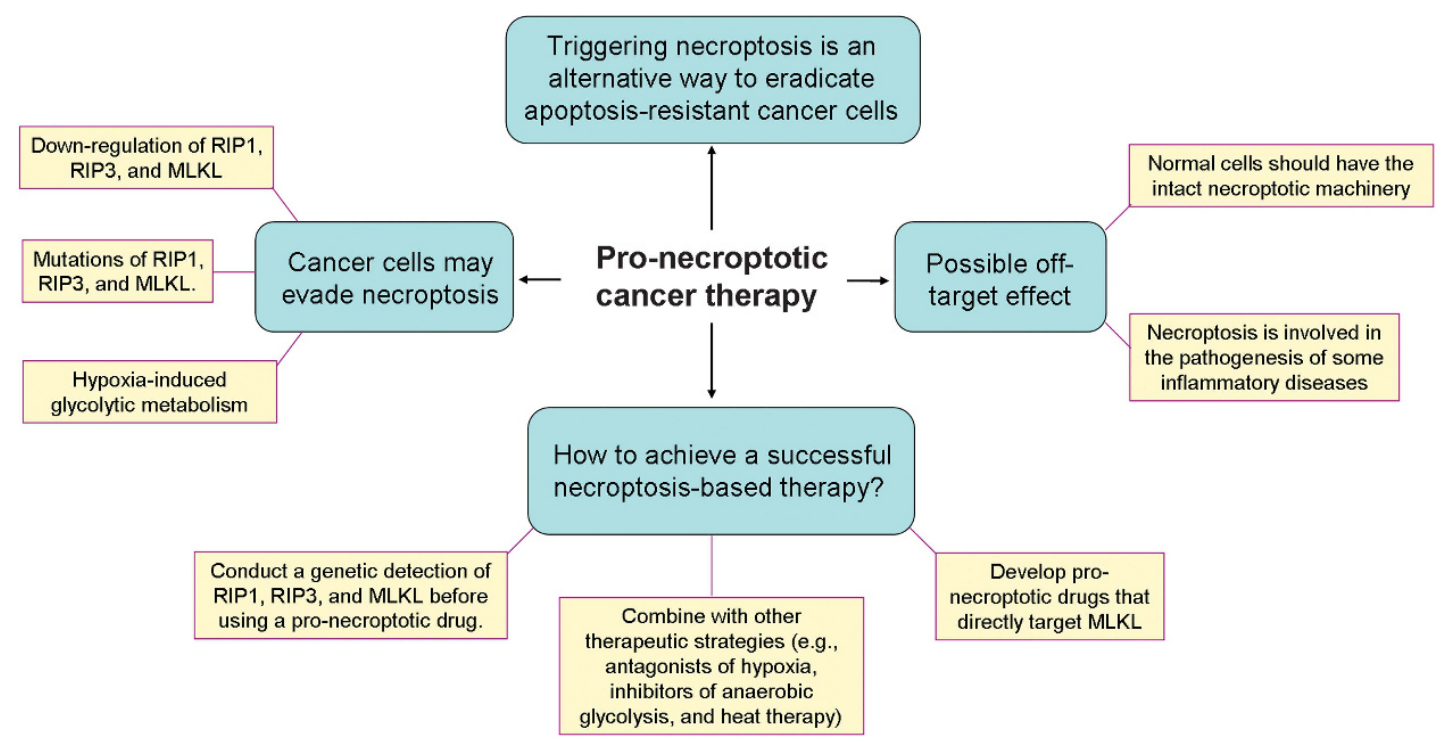

Figure 2 A diagram illustrating the pronecroptotic cancer therapy

but some compounds may bypass RIP1 to directly activate or inhibit RIP3, or even bypass RIP1 and RIP3 to activate or inhibit MLKL. For example, poly I:C can induce necroptosis in murine embryonic fibroblasts or human cervical cancer cells in a RIP1-independent manner; 6,46 necrosulfonamide can inhibit necroptosis by targeting MLKL directly in a RIP1- and RIP3-independent manner. ${ }^{34}$ As RIP1 and RIP3 expressions are inclined to be decreased because of genetic mutations or hypoxic induction, pharmaceutical companies may develop new pronecroptosis agents that directly target and activate MLKL. This could be a promising therapeutic strategy to overcome necroptosis resistance in cancer cells.

\section{Concerns About Pronecroptotic Therapy}

Specificity. As necroptosis is a phenomenon in many physiological and pathological processes such as virus infection and ischemic stroke, normal cells should have the intact necroptotic machinery. Therefore, the major concern is whether necroptosis inducers have a selectivity in triggering cancer cell death but not normal cell death. In fact, many traditional chemotherapeutic or molecular targeted drugs approved for marketing or in clinical trials recently have been identified as cancer necroptosis inducers in some cancer types (as shown in Table 1). These drugs are TRAIL, ${ }^{18}$ obatoclax alone, ${ }^{47}$ obatoclax plus dexamethasone, ${ }^{27} 3$ bromopyruvate plus chloroquine, ${ }^{48}$ and shikonin and its analogs. ${ }^{30,36}$ These drugs have been proven to be safe for human use; induction of necroptosis in cancer cells does not have to be toxic to normal cells and lead to severe side effects in vivo.

However, to further enhance the specificity and selectivity of pronecroptosis drugs, pharmaceuticals may consider conjugating necroptosis inducers to tumor-guided drugs (e.g. folate) or tumor-targeting antibodies.
Necroptosis-related inflammation. Rapid and massive release of damage-associated molecular patterns (e.g. IL-1 family cytokines, nucleic acids, ribonucleoproteins, histones, HMGB family members, and heat-shock proteins) from necroptotic cancer cells may act as strong inducers of inflammation. ${ }^{49}$ Numerous studies have suggested that inflammatory microenvironment may promote tumor development by stimulating angiogenesis and metastasis, attenuating adaptive immune responses, and increasing drug resistance. $^{50}$ Therefore, another concern is whether necroptosis-related inflammation has a deleterious role in tumor treatment. In addition, because necroptosis has been reported to be involved in the pathogenesis of some inflammatory diseases, such as inflammatory bowel disease, Crohn's disease, and inflammatory skin diseases, ${ }^{49}$ the potential off-target effect of necroptosis inducers may have the possibility to trigger pathological inflammation in normal tissues. These concerns should be explored and clarified in future in vivo experiments. Figure 2 is a diagram illustrating the pronecroptotic cancer therapy.

\section{Perspective}

As we have discussed in this review, certain cancer cells can undergo necroptosis upon certain physical or chemical stimuli. As the necroptotic biochemical pathway is distinct from the apoptotic pathway, triggering necroptosis could be a promising alternative strategy to overcome the resistance to proapoptotic chemotherapeutic agents. However, cancer cells also have evolved countermeasures to evade necroptosis by genetic mutations or downregulations of RIP1, RIP3, or MLKL. Hypoxic microenvironment also have an important role in conferring resistance to necroptosis induction. Owing to these intrinsic or acquired necroptosis resistance, screenings for gene mutations and aberrant protein expression are required before administering the necroptosis-based therapy. Moreover, other therapies, such as heat therapy and antihypoxia 
therapy, or pharmaceutical development of MLKL agonists used in combination with necroptotic cancer therapy could be beneficial in overcoming the resistance to necroptosis.

In addition, there are still some mechanisms that need further clarification. For example, it is known that radiation therapy can induce both necrosis and apoptosis in tumors, ${ }^{51,52}$ but whether radiation-induced necrosis is necroptosis is still under investigation. Nehs et al. ${ }^{53}$ reported that Nec-1 partially protected the radiation-induced cell death in anaplastic thyroid and adrenocortical cancer cells. This suggests that necroptosis may be involved in radiationrelated cancer therapy; however, more experiments should be conducted to confirm the necroptotic cell death.

So far, most studies on cancer and necroptosis are based on in vitro experiments, thus the efficacy of necroptosis inducer in vivo and the selectivity in killing tumors still need more investigation. For future studies, researchers should evaluate the off-target effects of necroptosis-based cancer therapy and develop strategies to enrich pronecroptosis drugs in tumors by conjugation with other tumor-guided drugs or antibodies. In addition, the potential inflammatory responses related to necroptosis induction should be taken into account and evaluated. Currently, the most well-known necroptosis inducers are not suitable for therapeutic purposes. Few research groups or companies have carried out necroptosisbased anticancer drug screens. It is necessary to develop more potent and effective necroptosis inducers that have a high specificity to cancer cells but low side effects in normal cells. Moreover, based on present studies, triggering necroptosis seems to be most effective in colorectal and hematopoietic cancer cells; therefore, it would be meaningful to build on these current findings to establish an effective cancer therapy regimen. In parallel, more research should be conducted to identify other tumors that are suitable for necroptosis-based therapy and to determine the underlying mechanisms.

\section{Conflict of Interest}

The authors declare no conflict of interest.

Acknowledgements. This research was supported, in part, by grants from the National Natural Science Foundation of China (Nos 81460440, 31300666, 81260322, 81372322 and 81560471), the China Postdoctoral Science Foundation (Nos 2013M542478 and 2015T81139), the Natural Science Foundation of Yunnan Province (No. 2012FB163), the Joint Special Funds for the Department of Science and Technology of Yunnan Province-Kunming Medical University (No. 2014FB059), the Scientific Research Projects from Internal Research Institutions of Medical and Health Units in Yunnan Province (Nos 2014NS013, 2014NS014, 2014NS015, and 2014NS016), Foundation of the Yunnan Provincial Innovative Team of Bone and Soft Tissue Tumor (No.2015HC026), Foundation of the Young and Middle-aged Academic and Technical Leaders of Yunnan Province (No.2014HB034), and Doctor Scientific Research Startup Funds of the Third Affiliated Hospital of Kunming Medical University (No. BSJJ201406).

1. Chan FK-M. Programmed necrosis/necroptosis: an inflammatory form of cell death. Cell Death. Springer, 2014, 211-228.

2. Berghe TV, Linkermann A, Jouan-Lanhouet S, Walczak H, Vandenabeele P. Regulated necrosis: the expanding network of non-apoptotic cell death pathways. Nat Rev Mol Cell Biol 2014; 15: 135-147.

3. Su Z, Yang Z, Xu Y, Chen Y, Yu Q. Apoptosis, autophagy, necroptosis, and cancer metastasis. Mol Cancer 2015; 14: 48
4. Jouan-Lanhouet S, Riquet F, Duprez L, Berghe TV, Takahashi N, Vandenabeele P. Necroptosis, in vivo detection in experimental disease models. Semin Cell Dev Biol 2014; 35: 2-13.

5. Cai Z, Jitkaew S, Zhao J, Chiang H-C, Choksi S, Liu J et al. Plasma membrane translocation of trimerized MLKL protein is required for TNF-induced necroptosis. Nat Cell Biol 2014; 16 : 55-65.

6. Dillon CP, Weinlich R, Rodriguez DA, Cripps JG, Quarato G, Gurung P et al. RIPK1 blocks early postnatal lethality mediated by caspase-8 and RIPK3. Cell 2014; 157: 1189-1202.

7. Christofferson DE, Yuan J. Necroptosis as an alternative form of programmed cell death. Curr Opin Cell Biol 2010; 22: 263-268.

8. Oberst A, Dillon CP, Weinlich R, McCormick LL, Fitzgerald P, Pop C et al. Catalytic activity of the caspase-8-FLIPL complex inhibits RIPK3-dependent necrosis. Nature 2011; 471 363-367.

9. Kaiser WJ, Upton JW, Long AB, Livingston-Rosanoff D, Daley-Bauer LP, Hakem R et al. RIP3 mediates the embryonic lethality of caspase-8-deficient mice. Nature 2011; 471 : 368-372.

10. Zhang H, Zhou X, McQuade T, Li J, FK-M Chan, Zhang J. Functional complementation between FADD and RIP1 in embryos and lymphocytes. Nature 2011; 471: 373-376.

11. Ch'en IL, Beisner DR, Degterev A, Lynch C, Yuan J, Hoffmann A et al. Antigen-mediated T cell expansion regulated by parallel pathways of death. Proc Natl Acad Sci USA 2008; 105: 17463-17468.

12. Bell $B D$, Leverrier $S$, Weist $B M$, Newton $R H$, Arechiga AF, Luhrs KA et al. FADD and caspase-8 control the outcome of autophagic signaling in proliferating T cells. Proc Natl Acad Sci USA 2008; 105: 16677-16682.

13. Lu JV, Weist BM, van Raam BJ, Marro BS, Nguyen LV, Srinivas $P$ et al. Complementary roles of Fas-associated death domain (FADD) and receptor interacting protein kinase-3 (RIPK3) in T-cell homeostasis and antiviral immunity. Proc Natl Acad Sci USA 2011; 108: 15312-15317.

14. Huang Z, Wu S-Q, Liang Y, Zhou X, Chen W, Li L et al. RIP1/RIP3 binding to HSV-1 ICP6 initiates necroptosis to restrict virus propagation in mice. Cell Host Microbe 2015; 17: 229-242.

15. Cho Y, Challa S, Moquin D, Genga R, Ray TD, Guildford M et al. Phosphorylation-driven assembly of the RIP1-RIP3 complex regulates programmed necrosis and virus-induced inflammation. Cell 2009; 137: 1112-1123.

16. Zhou W, Yuan J. Necroptosis in health and diseases. Semin Cell Dev Biol 2014; 35: 14-23.

17. Moriwaki K, Bertin J, Gough P, Orlowski G, Chan FK. Differential roles of RIPK1 and RIPK3 in TNF-induced necroptosis and chemotherapeutic agent-induced cell death. Cell Death Dis 2015; 6: e1636.

18. Meurette $O$, Rebillard A, Huc L, Le Moigne G, Merino D, Micheau $O$ et al. TRAlL induces receptor-interacting protein 1-dependent and caspase-dependent necrosis-like cell death under acidic extracellular conditions. Cancer Res 2007; 67: 218-226.

19. Jouan-Lanhouet S, Arshad M, Piquet-Pellorce C, Martin-Chouly C, Le Moigne-Muller G, Van Herreweghe $F$ et al. TRAIL induces necroptosis involving RIPK1/RIPK3-dependent PARP-1 activation. Cell Death Differ 2012; 19: 2003-2014.

20. Philipp S, Sosna J, Plenge J, Kalthoff H, Adam D. Homoharringtonine, a clinically approved anti-leukemia drug, sensitizes tumor cells for TRAIL-induced necroptosis. Cell Commun Signal 2015; 13: 25.

21. Huang C, Kuo W, Huang Y, Lee T, Yu L. Resistance to hypoxia-induced necroptosis is conferred by glycolytic pyruvate scavenging of mitochondrial superoxide in colorectal cancer cells. Cell Death Dis 2013; 4: e622.

22. Takemura R, Takaki H, Okada S, Shime H, Akazawa T, Oshiumi H et al. Poly I: C-induced, TLR3/RIP3-dependent necroptosis backs up immune effector-mediated tumor elimination in vivo. Cancer Immunol Res 2015; 3: 902-914.

23. Xie X, Zhao $Y$, Ma CY, Xu XM, Zhang YQ, Wang CG et al. Dimethyl fumarate induces necroptosis in colon cancer cells through glutathione depletion/ROS increase/MAPKs activation pathway. Br J Pharmacol 2015; 172: 3929-3943.

24. Metzig MO, Fuchs D, Tagscherer K, Gröne H, Schirmacher P, Roth W. Inhibition of caspases primes colon cancer cells for 5 -fluorouracil-induced TNF- $\alpha$-dependent necroptosis driven by RIP1 kinase and NF- $\kappa$ B. Oncogene 2015.

25. Degterev A, Hitomi J, Germscheid M, Ch'en IL, Korkina O, Teng X et al. Identification of RIP1 kinase as a specific cellular target of necrostatins. Nat Chem Biol 2008; 4: 313-321.

26. Yamanaka K, Urano Y, Takabe W, Saito Y, Noguchi N. Induction of apoptosis and necroptosis by $24(S)$-hydroxycholesterol is dependent on activity of acyl-CoA: cholesterol acyltransferase 1. Cell Death Dis 2014; 5 : e990.

27. Bonapace L, Bornhauser BC, Schmitz M, Cario G, Ziegler U, Niggli FK et al. Induction of autophagy-dependent necroptosis is required for childhood acute lymphoblastic leukemia cells to overcome glucocorticoid resistance. J Clin Invest 2010; 120: 1310.

28. Steinhart L, Belz K, Fulda S. Smac mimetic and demethylating agents synergistically trigger cell death in acute myeloid leukemia cells and overcome apoptosis resistance by inducing necroptosis. Cell Death Dis 2013; 4: e802.

29. Chromik J, Safferthal C, Serve H, Fulda S. Smac mimetic primes apoptosis-resistant acute myeloid leukaemia cells for cytarabine-induced cell death by triggering necroptosis. Cancer Lett 2014; 344: 101-109.

30. Xuan Y, Hu X. Naturally-occurring shikonin analogues - a class of necroptotic inducers that circumvent cancer drug resistance. Cancer Lett 2009; 274: 233-242.

31. Dunai ZA, Imre G, Barna G, Korcsmaros T, Petak I, Bauer PI et al. Staurosporine induces necroptotic cell death under caspase-compromised conditions in U937 cells. PLoS One 2012; 7: e41945. 
32. Wada N, Kawano Y, Fujiwara S, Kikukawa Y, Okuno Y, Tasaki M et al. Shikonin, dually functions as a proteasome inhibitor and a necroptosis inducer in multiple myeloma cells. Int $J$ Oncol 2015; 46: 963-972.

33. McCabe K, Bacos K, Lu D, Delaney J, Axelrod J, Potter M et al. Triggering necroptosis in cisplatin and IAP antagonist-resistant ovarian carcinoma. Cell Death Dis 2014; 5: e1496.

34. Wang Z, Jiang H, Chen S, Du F, Wang X. The mitochondrial phosphatase PGAM5 functions at the convergence point of multiple necrotic death pathways. Cell 2012; 148: 228-243.

35. Holohan C, Van Schaeybroeck S, Longley DB, Johnston PG. Cancer drug resistance: an evolving paradigm. Nat Rev Cancer 2013; 13: 714-726.

36. Han W, Li L, Qiu S, Lu Q, Pan Q, Gu Y et al. Shikonin circumvents cancer drug resistance by induction of a necroptotic death. Mol Cancer Ther 2007; 6: 1641-1649.

37. Laukens B, Jennewein C, Schenk B, Vanlangenakker N, Schier A, Cristofanon S et al. Smac mimetic bypasses apoptosis resistance in FADD-or caspase-8-deficient cells by priming for tumor necrosis factor $\alpha$-induced necroptosis. Neoplasia 2011; 13: 971-IN929.

38. Nugues A-L, El Bouazzati H, Hetuin D, Berthon C, Loyens A, Bertrand E et al. RIP3 is downregulated in human myeloid leukemia cells and modulates apoptosis and caspasemediated p65/RelA cleavage. Cell Death Dis 2014; 5: e1384.

39. Liu P, Xu B, Shen W, Zhu H, Wu W, Fu Y et al. Dysregulation of TNF $\alpha$-induced necroptotic signaling in chronic lymphocytic leukemia: suppression of CYLD gene by LEF1. Leukemia 2011; 26: 1293-1300

40. Colbert LE, Fisher SB, Hardy CW, Hall WA, Saka B, Shelton JW et al. Pronecrotic mixed lineage kinase domain-like protein expression is a prognostic biomarker in patients with early-stage resected pancreatic adenocarcinoma. Cancer 2013; 119: 3148-3155.

41. Fukasawa M, Kimura M, Morita S, Matsubara K, Yamanaka S, Endo C et al. Microarray analysis of promoter methylation in lung cancers. J Hum Genet 2006; 51: 368-374.

42. Forbes S, Bhamra G, Bamford S, Dawson E, Kok C, Clements J et al. The catalogue of somatic mutations in cancer (COSMIC). Curr Protoc Hum Genet 2008; Unit 10.11.

43. Cerhan JR, Ansell SM, Fredericksen ZS, Kay NE, Liebow M, Call TG et al. Genetic variation in 1253 immune and inflammation genes and risk of non-Hodgkin lymphoma. Blood 2007; 110: 4455-4463.

44. Wilson WR, Hay MP. Targeting hypoxia in cancer therapy. Nat Rev Cancer 2011; 11 393-410.

45. Mouratidis PX, Rivens I, ter Haar G. A study of thermal dose-induced autophagy, apoptosis and necroptosis in colon cancer cells. Int J Hypertherm 2015; 31: 476-488.

46. Schmidt SV, Seibert S, Walch-Rückheim B, Vicinus B, Kamionka E-M, Pahne-Zeppenfeld J et al. RIPK3 expression in cervical cancer cells is required for poly IC-induced necroptosis, $\mathrm{IL}-1 \alpha$ release, and efficient paracrine dendritic cell activation. Oncotarget 2015; 6 : 8635-8647.

47. Basit F, Cristofanon S, Fulda S. Obatoclax (GX15-070) triggers necroptosis by promoting the assembly of the necrosome on autophagosomal membranes. Cell Death Differ 2013; 20: 1161-1173.

48. Zhang $Q$, Zhang Y, Zhang P, Chao Z, Xia F, Jiang $C$ et al. Hexokinase II inhibitor, 3-BrPA induced autophagy by stimulating ROS formation in human breast cancer cells. Genes Cancer 2014; 5: 100.

49. Pasparakis M, Vandenabeele P. Necroptosis and its role in inflammation. Nature 2015; 517 311-320.

50. Mantovani A, Allavena P, Sica A, Balkwill F. Cancer-related inflammation. Nature 2008; 454 436-444.

51. Dewey WC, Ling CC, Meyn RE. Radiation-induced apoptosis: relevance to radiotherapy. Int J Radiat Oncol Biol Phys 1995; 33: 781-796.
52. Chernov M, Hayashi M, Izawa M, Ochiai T, Usukura M, Abe K et al. Differentiation of the radiationinduced necrosis and tumor recurrence after gamma knife radiosurgery for brain metastases: importance of multi-voxel proton MRS. Minim Invasive Neurosurg 2005; 48: 228-234.

53. Nehs MA, Lin C-I, Kozono DE, Whang EE, Cho NL, Zhu K et al. Necroptosis is a novel mechanism of radiation-induced cell death in anaplastic thyroid and adrenocortical cancers. Surgery 2011; 150: 1032-1039.

54. Bronner DN, O'Riordan MX, He Y. Caspase-2 mediates a Brucella abortus RB51-induced hybrid cell death having features of apoptosis and pyroptosis. Front Cell Infect Microbiol 2013; 3: 83.

55. Saddoughi SA, Gencer S, Peterson YK, Ward KE, Mukhopadhyay A, Oaks J et al. Sphingosine analogue drug FTY720 targets I2PP2A/SET and mediates lung tumour suppression via activation of PP2A-RIPK1-dependent necroptosis. EMBO Mol Med 2013; 5: 105-121.

56. Suntharalingam K, Awuah SG, Bruno PM, Johnstone TC, Wang F, Lin W et al. Necroptosisinducing rhenium (V) oxo complexes. J Am Chem Soc 2015; 137: 2967-2974.

57. He W, Wang Q, Srinivasan B, Xu J, Padilla M, Li Z et al. A JNK-mediated autophagy pathway that triggers C-IAP degradation and necroptosis for anticancer chemotherapy. Oncogene 2014; 33: 3004-3013.

58. Vanlangenakker N, Berghe TV, Bogaert P, Laukens B, Zobel K, Deshayes K et al. clAP1 and TAK1 protect cells from TNF-induced necrosis by preventing RIP1/RIP3-dependent reactive oxygen species production. Cell Death Differ 2011; 18: 656-665

59. Hitomi J, Christofferson DE, Ng A, Yao J, Degterev A, Xavier RJ et al. Identification of a molecular signaling network that regulates a cellular necrotic cell death pathway. Cell 2008; 135: 1311-1323.

60. Karl I, Jossberger-Werner M, Schmidt N, Horn S, Goebeler M, Leverkus M et al. TRAF2 inhibits TRAIL-and CD95L-induced apoptosis and necroptosis. Cell Death Dis 2014; 5: e1444.

61. Kim SK, Kim W-J, Yoon J-H, Ji J-H, Morgan MJ, Cho H et al. Upregulated RIP3 expression potentiates MLKL phosphorylation-mediated programmed necrosis in toxic epidermal necrolysis. J Invest Dermatol 2015; 135: 2021-2030.

62. Ratovitski EA. Phospho- $\Delta$ Np63 $\alpha$-responsive microRNAs contribute to the regulation of necroptosis in squamous cell carcinoma upon cisplatin exposure. FEBS Lett 2015; 589: 1352-1358.

63. Yu X, Deng Q, Li W, Xiao L, Luo X, Liu X et al. Neoalbaconol induces cell death through necroptosis by regulating RIPK-dependent autocrine TNF $\alpha$ and ROS production. Oncotarget 2014; 6: 1995-2008.

64. Nomura M, Ueno A, Saga K, Fukuzawa M, Kaneda Y. Accumulation of cytosolic calcium induces necroptotic cell death in human neuroblastoma. Cancer Res 2014; 74: 1056-1066.

65. Coupienne I, Fettweis G, Rubio N, Agostinis P, Piette J. 5-ALA-PDT induces RIP3dependent necrosis in glioblastoma. Photochem Photobiol Sci 2011; 10: 1868-1878.

(c) (i) $(9)$ This work is licensed under a Creative Commons Attribution-NonCommercial-NoDerivs 4.0 International License. The images or other third party material in this article are included in the article's Creative Commons license, unless indicated otherwise in the credit line; if the material is not included under the Creative Commons license, users will need to obtain permission from the license holder to reproduce the material. To view a copy of this license, visit http://creativecommons.org/licenses/by-nc-nd/4.0/ 